VII.

Aus der chirurgischen Abteilung des Altonaer Stadtkrankenhauses. (Chefarzt: Prof. Dr. König.)

\title{
Ein Beitrag zur Frage der Contagiosität des Erysipels.
}

\author{
Yon \\ 1)r. Franke.
}

Die Lehre von der Contagiosität des Frysipels ist zu alt, als daß sie sich in kurzer Zeit durch Arbeiten ausrotten ließe, die unter Zuhilfenahme aller modernen bakteriologischen Untersuchungsmethoden das Gegenteil nachzuweisen suchen. Noch immer ist der Respekt vor einer allzu nahen Berührung des an Erysipel Frkrankten so groß, daß im stationären wie poliklinischen Dienst der damit Behaftete wie ein Verpesteter gemieden und zurückgewiesen wird, bis alle sonstige Arbeit getan ist. Und dabei läßt es sich in einer größeren Poliklinik nie vermeiden, daß man nach Eröffnung phlegmonöser oder sonst eitriger Prozesse, bei denen die Hand des Operateurs mit voll virulenten Bakterien inficiert wurde, gezwungen ist, eine ganz aseptische Operation vorzunehmen. Warum nur wird das Erysipel so scharf von anderen, durch gleiche Bakterienarten hervorgerufene Erkrankungen getrennt?

Allgemein anerkanut ist wohl die Lehre, daß stets ein Epitheldefekt in Hant oder Schleimhaut vorhanden sein muß, durch den die Erreger ins subkutane Gewebe dringen können. Damit ist eigentlich schon festgestellt, daß das Erysipel eine Wundinfektionskrankheit ist. Dieser möchte ich die Krankheiten als contagiös gegenüberstellen, die durch ein flüchtiges, hochgradig virulentes, im vulgären Sinne ansteckendes Contagium erzeugt werden. Hier ist es möglich, daß die Krankheitserreger durch Zwischenträger oder durch die Luft über weite Strecken fort an ein gerade für sie empfängliches Individuum geraten und dies krank machen. Jer dem Worte Contagium zugrunde liegende Begriff Contakt darf uns hier 
nicht irreführen, da er außerordentlich vieldeutig ist. In den letzten Jahren haben einige Autoren nachzuweisen versucht, daß eine solche Contagiosität beim Erysipel nicht vorliegt. Allerdings ist die Zahl dieser Arbeiten noch klein, und da immerhin diese Frage für unsere Auffassung und unser ganzes Handeln bestimmend ist, so dürfte ich vielleicht berechtigt sein, auf Grund einiger Untersuchungen dies Thema wieder einmal aufzunehmen.

Das Erysipel hat ja längst aufgehört ein Schrecken der chirurgischen Abteilungen zu sein, die einst von förmlichen Epidemien heimgesucht wurden. Wieviel von diesem Erfolge auf Rechnung: unserer besseren Kenntnisse über Wundbehandlung und Wundinfektionen zu setzen ist, wieviel auf eine exaktere Ausbildung und Controllierung des Personals, wieviel endlich auf eine schärfere Diagnosenstellung, darauf will ich hier nicht weiter eingehen, sicher ist, daß die Berichte über Erysipel-Epidemien nahezu aus unserer Literatur verschwunden sind. Gerade aus diesen Epidemien zogen die früheren Autoren den begreiflichen Schluß, daß ein flüchtiges Contagium die Krankheit vermitteln mïsse, ähnlich wie wir es z. B. von den Morbillen annehmen. Diese Beweisführung ist gewiß anzufechten, denn wir haben an einer genügend großen Anzahl von früher contagiösen Krankheiten mit aller Sicherheit nachgewiesen, dals sie infektiös sind. Doch anch trotz Fortfalles jener epidemischen Heimsuchungen sind die immer noch reichlich genug auftretenden sporadischen Fälle geeignet, die Verzweiflung des Stationsarztes, speziell aber des Chirurgen wachzurufen, denn er muß ja bei einem einzigen auf der Station ausgebrochenen Erysipel - vorausgesetzt, daß es sich um ein flüchtiges Contagium handelt - für seine sämtlichen anderen aseptisch Operierten fürchten. Die geheimnisvolle Art, wie das Erysipel solche Einzelattacken ausübt, die in vielen Fällen bestehende Unmöglichkeit des Nachweises der Eingangspforte und der Herkunft der Entzündungserreger können unsere Scheu nur steigern.

Der letzte vereinzelte Bericht über eine Epidemie von Erysipel, soweit ich übersehen kann, stammt von Uche. Im Winter 1894/95 kamen im Militärhospital in Warschau in 8 Monaten 213 Erkrankungen vor. Im Anschluß hieran untersuchte der Autor den Staub von Krankensälen und fand in 10 von 69 Fällen Streptokokken. Ausführlich schildert er, wie er in dem Staube eines kleinen, vor einer Ventilationsöffuung angebrachten Drahtnetzes, das dicht an einem Bette stand, in welchem schon wiederholt Erysipel zum Ausbruch gekommen war, für Kaninchen virulente Streptokokken fand. 
Ähnliches haben auch andere Untersucher, v. Eiselsberg, Emmerich u. a., nachgewiesen, und gerade die beiden angeführten Autoren fanden nicht nur im Staube von Krankensälen, sondern auch in der Luft Streptokokken, allerdings sind diese positiven Befunde sehr rar, und es fehlen dabei Angaben über die Virulenz. Gesetzt nun den Fall, alle diese im Staube gefundenen Streptokokken seien virulent und könnten in Wunden ein Erysipel erzeugen, so ist mit dieser Kenntnis noch nichts für die eventuelle Gefährlich. keit des nun Erkrankten gewonnen. Alles drängt sich daher auf die Frage zusammen, ob virulente Streptokokken den an Erysipel erkrankten Körper verlassen können oder nicht.

In richtiger Erwägung dieser Gefahr hat man von jeher ein Hauptaugenmerk auf die Schuppen gerichtet. Jedem Beobachter drängte sich natürlich der Vergleich mit Scarlatina anf und nichts lag näher, als die Erfahrungen der einen auf die andere Erkrankung: zu übertragen. Jedoch ist dies nicht der einzige Weg, auf dem die Streptokokken den Körper verlassen könnten, sondern hier kommen noch der Schweiß und beim Schleimhauterysipel das spez. Sekret, so beim Erysipelas faciei das Nasensekret in Betracht. Endlich wären noch beim Erysipelas bullosum das Exsudat in den Blasen zu berücksichtigen, die beim Platzen etwa enthaltene Kokken an ihre Umgebung weiter geben können. Drei Autoren haben sich mit der Untersuchung. der Schuppen näher befaßt, v. Eiselsberg, Achalme und Respinger. v. Eiselsberg vermochte bei 5 Patienten 4 mal Streptokokken in den Schuppen nachzuweisen, die in 2 Fällen für Kaninchen virulent waren. Achalme*) berichtet über 22 Fälle, bei denen er den Bakteriengehalt der Schuppen sowohl in Bouillon als mittelst Agarplatten untersuchte und kein einziges Mal Streptokokken, sondern nur Staphylokokken und Bazillen finden konnte. Respinger untersuchte sehr genau 17 Fälle von Erysipel und vermochte nie in den Schuppen Streptokokken nachzuweisen. Nur wenn er direkt neben der Infektionspforte die Schuppen entnahm, hatte er ein positives Resultat in einigen Fällen, und er glaubt, daß auch die positiven Ergebnisse von v. Eiselsberg darauf zurückzuführen seien, daß die Entnahme der Schuppen nahe der Wunde, also an einer Stelle geschehen sei, wo eine Verunreinigung von außen durch Wundsekret sehr leicht sei. Es gelang ihm auch in seinen positiven Fällen im Wundsekret Streptokokken nachzuweisen. Den wenigen, kritisch nicht ganz einwandsfreien Fällen

*) Cit. nach Respinger. 
mit positivem Ergebnis stehen also zahlreiche exakt untersuchte Fälle gegenüber, die die völlige Gefahrlosigkeit der Hautschuppen nachweisen.

In mikroskopischen Hautschnitten sind oft genug schon die Streptokokken in den Lymphräumen das Corium nachgewiesen, es liegt daher der Gedanke nahe, daß sie von hier aus in die Schweißdrüsen geraten können und beim Schwitzen an die Hautoberfläche gelangen. Es ist jedoch von mehreren Autoren (Suderkow, v. Noorden, Petruschky, Achalme, Respinger, Hägler) nachgewiesen, daß beim einfachen Erysipel keine Streptokokken im Schweiß sind, erst wenn die Kokken ins Blut übergehen und zur Allgemeininfektion führen, werden sie mit dem Blut auch den Schweißdrüsen zugeführt und sind dann im Schweiß vorhanden.

Gefährlicher als der Schweiß erscheint sicher der Blaseninhalt beim Erysipelas bullosum. Die Untersuchungen dieses Sekretes sind infolge dessen verhältnismäßig zahlreich. Trotzdem war nur v. Eiselsberg so glücklich, in 1 von 3 Fällen im Sekret einer bereits älteren, getrübten Blase außer Staphylokokken und Hautschmarozern Streptokokken zu zählen, mit denen er bei 7 von 8 Kaninchen am Ohr Erysipel erzeugte. Tillmanns machte, ehe man bakteriologisch den Streptococcus gefunden hatte, Impfversuche mit Blaseninhalt, und in 2 Fällen will er ein Erysipel erzeugt haben unter zahlreichen negativ ausfallenden Impfungen. Sicher beweisend sind diese 2 Fälle nicht, denn es fehlt der bakteriologische Nachweis des Streptococcus, zumal in neuerer Zeit Jordan über eine Beobachtung berichtet, wo er durch Staphylokokken allein Erysipel erzeugte. Fehleisen, der als erster bakteriologische Untersuchungen des Blaseninhalts anstellte, fand unter vielen ergebnislosen Versuchen einmal einen Streptococcus, der jedoch eine so geringe Virulenz zeigte, daß er beim Kultivieren abstarb. Respinger gibt dann außer seinen eigenen ganz negativen Fällen einen kurzen Bericht über 22 Fälle von Hägler, die alle bis auf einen ohne Streptokokken im Blasensekret waren, und dieser eine endete letal. Er läßt sich also kritisch nicht verwerten, da bei Allgemeininfektion natürlich Streptokokken ins Blut und in die Blasen gelangen können. Es bleibt also von so vielen Versuchen als einziger der von v. Eiselsberg mit positivem Streptokokkenbefund und er läßt sich allerdings nicht leicht erklären. Auffallend scheint mir nur, daß es schon eine ältere Blase war, daß neben zahlreichen Kolonien von Staphylokokken und anderen Bazillen nur ein mühsam zu isolierender Streptokokkenstamm gefunden wurde. Ausgeschlossen ist gewiß nicht, daß dieser 
eine Keim sich aus der nahe befindlichen, secernierenden Wunde auf die Blase verirrt hat und so bei der Impfung mitgenommen wurde. Wenn man bei diesen Befunden des Blaseninhalts zurückblickt auf die Untersuchungen über Streptokokkengehalt der Schuppen, so erscheint es gewiß erklärlich, daß ihre Untersuchung so ergebnislos war, da ja der Weg bis zu ihnen noch weiter ist.

Immerhin zeigt diese Literaturübersicht, daß eine einheitliche Meinung noch nicht vorhanden ist, sondern daß die Ergebnisse des einen Untersuchers denen des anderen widersprechen, oder doch wenigstens zu widersprechen scheinen.

Ehe ich nun näher auf meine Untersuchungen und deren Resultate eingehe, möchte ich einige Worte über meine Versuchsanordnung vorausschicken. Es kam mir nicht allein darauf an nachzuweisen, daß die Schuppen nach Ablauf des Erysipels keine Infektionserreger mehr bergen, sondern mir lag daran zu untersuchen, ob vom Ausbruch des Frysipels an bis zur völligen Restitutio ad intergrum der Erkrankte eine Gefahr für seine Umgebung bedeutet, ob sich also auf seiner Haut und in der ihn umgebenden Luft Streptokokken nachweisen lassen. Als bekannt und sicher festgestellt setze ich dabei das Vorkommen der Streptokokken im Staub voraus, so daß ich hierauf nicht eingegangen bin. Fis sind fast nur schwere, lang dauernde Frysipele, die ich berücksichtigt habe, besonders auch solche mit schneller Blasenbildung, da die stärkere Virulenz mir eventuell den Bakteriennachweis zu erleichtern schien. Dabei wählte ich zur Untersuchung möglichst die Randpartie des erkrankten Teiles, jedenfalls hielt ich mich von der Eingangspforte immer so fern, daß eine Beschmutzung dieser Stelle von der Wunde aus nicht anzunehmen war.

Sobald mir ein für meine Untersuchungen geejgnetes Erysipel gemeldet war, machte ich meine Impfungen in der Weise, daß ich erst mit einem sterilen Tupfer eine erkrankte Hautstelle energisch abrieb, dann kratzte ich mit einer sterilisierten Pincette an einer anderen die oberflächlichsten Hautschichten ab und schabte an einer dritten mit sterilem Skalpell leicht die Epidermis ab, ohne das Corium zu verletzen. In meinen letzten Versuchen skarificierte ich außerdem die Haut an einigen Stellen und impfte mit dem blutigserösen Sekret, um auf diese Weise noch eine Art Kontrolle auszuüben. Eventuell vorhandene, mit klar serösem Inhalt angefüllte Blasen wurden mit ausgeglühter Platinöse eröffnet und ihr Inhalt in gleicher Weise geimpft. Sobald die Entzündung abgelaufen war und das Schuppungsstadium einsetzte, wurden - immer in respekt- 
voller Entfernung von der Wunde - diese Schuppen abgezogen und verimptt. Jede Impfung wurde doppelt gemacht, indem ich einmal in Bouillon impfte und die hier wachsenden Bakterien später in Agar- und Gelatineplattenkulturen weiter verarbeitete, und indem ich zweitens gleich von demselben Material eine Agarplatte anlegte. Die Bouillonkulturen wurden vor ilirer Verarbeitung zu Platten mikroskopisch nach 24 und 4 S Stunden im gefärbten Präparat untersucht, jede auf den Platten gefundene Art mikroskopiert und jede einigermalsen verdächtige Einzelkultur wieder in Bouillon übertragen und nach 24 Stunden im Deckglaspräparat untersucht.

Außerdem stellte ich nebenher eine Reihe von Luftuntersuchungen an. Es wurde, als icl meine Untersuchungen begann, jeder Erysipelfall unserer Abteilung in ein nur für diesen Zweck reserviertes Zimmer verlegt, daher mußte die Luft dieses nur für 2 Betten bestimmten Raumes für meine Zwecke recht geeignet sein. Ich ließ3, eine Stunde bevor ich meine Agarplatten aufstellte, das Zimmer abschließen, damit nicht durch Hin- und Hergehen unnötig Staub aufgewirbelt würde. Dann stellte ich meine Platten auf unmittelbar neben dem Bett des Patienten und liels sie eine Viertelstunde offen. Dieser Verfahren wiederholte ich im Schuppungsstadium; obwohl ich durch die Untersuchung der Schuppen allein schon über deren etwaige Infektiosität Kenntnis erhalten konnte, schien mir es in Anbetracht der r. Eiselsbergschen Befunde doch wichtig genug, beide Verfahren einzuschlagen. Hierbei leitete mich anch nicht die Absicht, die Angaben anderer Autoren über den Keimgehalt der Luft, speziell den Gehalt an Streptokokken nachzuprïfen, da einerseits das Plattenverfahren hierzu nicht ausreicht und es andererseits wohl feststeht, dals beim Hin- und Herlaufen in den Krankensälen Staub hochgewirbelt wird und die neben anderen Entzündungserregern in ihm anch vorhandenen Streptokokken in die Luft geraten können. Mir lag daran zu sehen, ob der Erysipelatöse bei stinen Bewegungen im Bett von sich aus Streptokokken rerbreitet, und darum lieb ich erst eine Zeitlang das Zimmer schlieljen, ele ich meine Platten aufstellte.

In dieser Weise habe ich 10 Fälle von Erysipel untersucht.

1. A. M., 20 Jahre, am 22. 3. 03 wegen Fistula ani operiert; es besteht sehr starker Vaginalfluor. Am 25. 3. 03 erfolgt unter Schüttelfrost der Ausbruch eines Erysipelas bullosum mit handtellergroBen Blasen, das vom Anus aus die Glutäalgegend bis zum Kreuzhein und nach unten zu bis zur Mitte der Oberschenkel zieht. 
26. März. a) Von der Hautoberfläche: Staphylococcus aureus, Bacillus subtilis, Bacterium coli. Zahllose Kolonien.

b) Von einer Blase: steril.

c) Nach 3 Tagen von einer mit weiRgelblichem, dickflüssigem Inhalt gefüllten Blase: Staphylococcus aureus. aureus.

d) Aus der Wunde: Bacterium coli, Sarcina lutea, Staphylococcus

e) Vaginalsekret: Eine Flora der verschiedensten Keime, keine Streptokokken.

f) 1 Gelatine, 2 Agarplatten 15 Minuten an der Luft: Staphylococcus aureus, Bacillus subtilis, Sarcina lutea et alba, mehrere Schimmelpilze.

10. April. Schuppungsstadium. Es werden Schuppen vom After, von der Glutäalgegend, von den Oberschenkeln genommen. Zugleich wurden 3 Agarplatten rler Luft ausgesetzt, doch fanden sich keine Streptokokken, vorwiegend Staphylokokken, daneben Bacillus subtilis, große Diplokokken, Sarcine. - Keine Streptokokken.

Mit dem aus der Blase gezüchteten Staphylococcus impfte ich ein Kaninchenohr, es entstand eine kleine Verhärtung mit circumscripter Rötung, kein Erysipel.

2. A. T., 35 Jahre. Patientin war in stationärer Behandlung wegen Fractura colli femoris, die schließlich genäht wurde. Während sonst alles geheilt war, kam es von einer kleinen tamponiert gewesenen, granulierenden Stelle aus zu einen Erysipel am 2. 10. 03, das schnell uber das ganze linke Bein hinzog.

3. Okt. a) Von der Haut aus: Bacillus subtilis, große Diplokokken, große Mikrokokken, Staphylococcus albus.

b) Von einer Blase: steril.

c) Von der Wunde: Staphylococcus allsus.

d) 3 Gelatineplatten 15 Minuten an der Luft: Zahllose, gröBtenteils verflüssigende Keime.

13. Okt. Schuppungsstadium: Staphylococcus aureus, Sarcina lutea, große, dicke Stübchen; in der Luft gleichfalls keine Streptokoklien.

3. H. H., 11/2 Jahre. Tulerkulöse Fistel am linken Oberschenkel nahe dem Hüftgelenk. Der kleine Patient liegt schon längere Zeit auf derselben Station wie der vorige Fall. Am gleichen Tage, dem 2. Okt. 03, bricht von der Fistel ausgehend ein Erysipel aus, das den Bauch, das Gesäß und das ganze linke Bein befällt.

3. Okt. a) Von der Haut: Staphylococcus aureus, Bacterium coli Sarcina lutea.

b) Auf 3 Agarplatten 15 Minuten an der Iuft: Zahlreiche Kolonien von Sarcine, Staphylokokken etc.

c) Von der Wunde: Das rein seröse Sekret enthält keine Keime.

10. Okt. Schuppungsstadium: Die Schuppen enthalten besonders Staphylococcus aureus. Auf den Platten wie sonst. Keine Streptokokken.

4. A. K., 19 Jahre. Am 15. Okt. 03 aufgenommen wegen Parulis am rechten Unterkiefer, in deren Eiter sich Staphylococcus aureus und Micrococcus tetragenus finden. Am 20. Okt. bricht ein Erysipel aus, das von der Wunde aus den ganzen Kopf uberzieht. 
21. Okt. a) Impfung des eitrig-serösen Wundsehretes: Staplylococcus aureus.

b) Von rler Umgebung der Wunde: Staphylococeus aureus, grofie Diplnkokken, Microcoecus tetragenus.

c) Vom Rande des Erysipels: Die gleichen heime wie sonst.

d) 3 Gelatineplatten 15 Yinuten exponiert: Schimmelpilze, Staphylococcus aureus, mehrere Sarcinearten.

e) Kaninchenohr mit dem Wundsekret selhst geimpft. Es tritt eine geringe entzündliche Neigung in der Umgebung der Impfstelle ein. kein Eryipel, in wenigen Tagen ist alles verschwunden und nur eine etwa erbsengrobe Infiltration bleibt bestehen. Das gleiche Resultat ergibt eine Impfung mit dem aus der Umgebung der Wunde gezüchteten Bakterienremisch.

8. November. Schuppung-stadium. Weder ans den Schuppen, noch von den Platten lassen sich Streptokokken zuchten, es wachsen ähnliche Keime wie früher bei diesen Versuchen. Das Wundsekret enthält immer noch Staphylncoccus aureus. - Keine Streptokokken.

5. A. M., 29 Jahre. Patientin wurde am 5. 11.08 in moribundem Zustande, delirierend und benommen eingeliefert. Über die Anamnese ließ sich nichts in Erfahrung bringen. Es bestand ein beide Wangen, Augen und Stirn überziehendes Frysipel, phlegmonöse Infiltration der rechten Orbita mit eitriger Thrombophlebitis der größeren Venen. Lis wurde durch Spaltung eine Druckentlastung versucht, doch war das Bild der Meningitis bereits so ausgesprochen, dab an ein radikales Vorgehen nicht mehr zu denken war. Die Pationtin starb in der Yacht, die Sektion ergab ausgedehnte basilare eitrige Meningitis.

5. Xovember. a) Von der Ifaut: Staphylococcus aureus et albus, grobe Mikrokokken, Bacillus subtili:.

b) Impfung des bei der Operation sich ergebenden Ölems der Orbita, sowie der Thromben. Aus beiden: Streptokokien in Reinkultur, die beim Kaninchenohr ein mit 'T'mperaturerhöhung einhergehendes Erysipel hervorriefen, das lis zum Nacken reichte.

c) Implung des hei der Sektion sich ergehenden Eiters der Meningen: Streptococeus in Rrinkultur.

d) 3 Agarplatten 15 Minuten geöffnet an der Iuft ergeben kein anderes Revultat als fraher.

Es wurden Streptokokken nur im entzündlichen Gewebe, resp. in Eiter gretunden, sonst nicht.

6. I. Sch., 583 Jahre. Patientin leidet schon lange an einem uber handtellergrofen Ulcus des linken Unterschenkels, von dem schon mehrmals Frysipele ausgegangen sind. Einige Tage vor ihrer Lufnahme ist es von neuem zu einem Erysipel von hier aus grekommen, das bis zur IIüfte hinaufrieht.

Im 15. November 03 wirl sie aufgenommen, die Rötung des Unterschenkels ist nur noch gering, am Oberschenkel jedoch lebhaft. Luterschenlel stark teigig geschwollen.

16. November. a) Von der Haut: Sarcina lutea, grofie Mikrokokken, Bacillus subtilis, cin schimmelpilz. 
b) Von dem Ulcus, das eitrig belegt ist: Staphylococcus aureus et albus, lange dicke, unbewegliche Stäbchen, Streptococcus. Die Plattenkultur ist mit Keimen förmlich übersät. früher.

c) 3 Agarplatten 15 Ninuten an der Luft: Ähnliche Keime wie

28. November. An beiden Malleolen und der Wade haben sich Abszesse gebildet, die incidiert werden. Im Eiter finden sich: Staphylococcus aureus und Streptococcus.

Impfung mit dem Streptococcus am Kaninchenohr ergab ein Erysipel von geringer Intensität.

Die mit den inzwischen aufgetretenen Schuppen angelegten Kulturen enthielten fern von den Wunden keine Streptokokken, sondern nur harmlose Schmarotzer.

Streptokokken fanden sich in der Wunde und im Eiter.

7. S. Sch., 25 Jahre. 24. 1. 05 aufgenommen wegen Erysipelas faciei, das am linken Auge begonnen hat und in langsamem Fortschreiten den ganzen Kopf überzieht.

25. Jan. a) Von der Ilaut: Staphylococcus aureus, Bacillus subtilis, Micrococeus tetragenus.

b) Haut mit Messer geritzt und Impfung des blutig-serösen Sekrets: Auf der Agarplatte bilden sich 65 Kolonien hauptsächlich von Staphylococcus aureus, keine Streptokokken. In Bouillon jedoch konnte ich neben anderen Keimen einen Streptococcus zuchten, der am Kaninchenohr eine etwa 2 Tage anhaltende Rötung der Haut und stärkere GefäBfulllung des Ohres hervorrief, jedoch kein eigentliches Erysipel.

c) 3 Gelatineplatten 15 Minuten unbedeckt enthalten zahlreiche Keime, keine Streptokokken.

2. Febr. Es wird ein Lidabszeß am linken unteren Augenlid eröffnet. Der Eiter enthält neben Stapllylococcus aureus und albus auch Streptolrokken bei der Züchtung, die am Kaninchenohr eine ähnliche Reaktion wie oben hervorrufen.

Gleichzeitig werden von der linken Stirnseite Schuppen entnommen, die keine Streptokokken ergeben, desgleichen nicht die jetzt exponierten Agarplatten. Haut.

Streptokokken waren nur im Eiter und im Impfstich der

8. F. P., 20 Jahre. 26. 1. 05. Vorgestern ist ron der Nase aus ein Erysipel ausgebrochen, las mit starker Blasenbildung einhergeht und das ganze Gesicht und den Hals tiberzieht.

27. Jan. a) Von der Haut werden ähnliche Keime wie früher gezüchtet, keine Streptokokken.

b) Impfstich der Haut: Zahlreiche Kolonien, besonders von Staphylococcus aureus, lieine Streptokokken.

c) Impfung aus drei Hautblasen steril.

d) Drei Ararplatten, 15 Minuten exponiert, enthalten zahlreiche saprophytische Keime, auch Staphylokokken, keine Streptokokken.

6. Febr. Am rechten oberen Augenlid hat sich ein AbsceB gebildet, dessen Eiter neben Staphylococcus aureus auch Streptococcus beim Zủchten 
ergibt, doch kommt es am Kaninchenohr nicht zu einem Erysipel bei der Impfung mit dem Streptococcus.

Auch die Impfung der Schuppen von der Stirn, Ohren und Kinn ergibt ebensowenig wie die jetzt exponierten Agarplatten Streptokokken.

Es wurden also Streptokoken nur im Eiter gefunden.

9. A. L., 32 Jahre. Erysipel am rechten Arm, das von einer kleinen Operationswunde der Hand ausgeht, die aseptisch angelegt war, um das nach Puerperalfieber metastatisch erkrankte cariöse Os capitatum zu entfernen. Nach gutem Verlauf und bis auf eine kleine tamponiert gewesene Stelle primärer Heilung kommt es nach 16 Tagen, als 4 Tage zum Zwecke besserer Mobilisierung eine Stauungsbinde angelegt war, zu einem Erysipel. Keime.

16. März 05. a) In der oberflächlich granulierenden Wande lieine

b) Von der erysipelatösen Haut die gewöhnlichen Schmarotzer.

c) Impfstich der Haut: Zahlreiche Keime, Staphylococeus aureus etc.

29. März. Impfung der Hautschuppen ergibt ein ähnliches Resultat wie früher.

Streptokokken wurden nie gefunden.

10. R. L., 20 Jahre. Wegen Bubo inguinalis sinister operiert, von hier aus entsteht am 14. 3. 05 ein das linke Bein überziehendes Erysipelas bullosum.

16. März. a) In der Wunde: Staphylococcus aureus und Streptococcus, der am Kaninchenohr kein Erysipel erzeugt.

b) Im Inhalt von zwei Blasen vom Oberschenkel und Unterschenkel werden Keime nicht gefunden.

c) Von der Haut: Staphylococcus albus, Bacillus subtilis etc.

d) Impfstich der Haut: Neben Staphylococcus aureus und verschiedenen anderen Keimen Streptokokken, die beim Weiterzüchten absterben. kokken.

e) In 3 Agarglatten, 15 Minuten der Luft ausgesetzt, keine Strepto-

f) Impfung der Sehuppen: Staphylococcus albus, Bac. subtilis, Sarcina lutea, Diplokokken.

Streptokokken wurden also nur in der Wunde und im Impfstich der Haut gefunden.

Ehe ich auf die Resultate meiner Untersuchungen eingehe, möchte ich erwähnen, daß ich die Frage der Spezifität der Erysipelerreger nicht berührt habe, da ich glaube, daß sie trotz der jüngsten gegenteiligen Behauptung Jordans durch andere Autoren, vor allem Petruschky, im Sinne der Gleichwertigkeit der bei verschiedenen Krankheitsprozeßen gefundenen Streptokokken entschieden ist. Wenigstens steht die Angabe Jordans, der mit einem Staphylococcus einen dem Erysipel täuschend ähnlichen Krankheitsprozeß erzeugte, recht vereinzelt da. Meinen Fall 4 möchte ich nicht in diesem Sinne verwerten, obwohl ich weder im Eiter noch später nach Ausbruch des Erysipels im Wundsekret Strepto- 
kokken fand. Da die Mundhöhle mit dem Abszeß in Verbindung stand, so läßt sich ein Hineingelangen von Streptokokken in die Wunde vom Munde aus leicht erklären. Außerdem aber spricht der negative Ausfall der Kaninchenohrimpfung mit dem Eiter sowohl, wie mit dem Bakteriengemisch gegen die Annahme, daß das Erysipel etwa durch Staphylokokken hervorgerufen sei. Nur ein Streptococcus kann Erysipel erzeugen, wobei seine Herkunft gleichgültig ist. Gerade dies letztere aber ist außerordentlich wichtig, da jeder Kranke mit einer streptomykotischen Eiterung eine dauernde Quelle der Infektion für sich und seine Umgebung mit sich herumträgt. Daher ist es begreiflich, wenn Klemm auf Grund seiner Untersuchungen die Forderung anfstellt, daß jeder Kranke mit einer Streptokokkeninfektion, ganz gleich welcher Provenienz sie ist, von den übrigen Kranken isoliert werden soll. Er läßt jede frisch aufgenommene Eiterung sofort bakteriologisch untersuchen und sondert auf Grund des bakteriologischen Befundes in solche ohne und solche mit Streptokokkeninfektion. Letztere werden sämtlich auf einer eigenen, für diesen Zweck reservierten Abteiling untergebracht.

Die Ergebnisse meiner Arbeit stehen im Einklang mit den Untersuchungen von Klemm, Respinger und Achalme. Der Streptococcus dringt durch eine unbedeutende, oft nicht mehr auffindbare Haut- oder Schleimhautverletzung und wächst in den Lymphbahnen der Cutis weiter, so daß das Erysipel eine exquisit lymphangoitische Erkrankung ist. Niemals aber kommt es vor, daß aus der Tiefe dieses Gewebes der Streptococcus an die Oberfläche gelangt und hier in Blasen oder Hautschuppen nachzuweisen wäre. $\mathrm{Ob}$ er nun - einmal ins Gewebe geraten - zum Erysipel führt oder zur Phlegmone, das hängt vielleicht $a b$ von der Größe der contundierenden Wunde, also anch von der Tiefe, in die er gelangt, dann aber auch wohl von der verschiedenen Virulenz, die es auch erklärt, warum er beim Kaninchen das eine Mal zum Abszeß, das andere Mal zum Erysipel führt.

Da es in mikroskopischen Schnitten erysipelatöser Haut nie gelang, Bakterien im Epithel oder zwischen den Epithelzellen des Rete Malpighi nachzuweisen, sie aber stets in den Lymphräumen der Cutis gefunden wurden, so wäre auch gar nicht $\mathrm{zu}$ verstehen, wie die Streptokokken von der Cutis in das gesunde, fest gefügte Epithelgewebe gelangen sollten. Am verständlichsten scheint das noch beim Erysipelas bullosum, das Exsudat könnte beim Filtrieren durch die sonst engen Maschen sie erweitern und infolge des 
starken Druckes dann Kokken mitgehen heilien. In diesem Fille möchte man abel rerlangen, dals sofort nach dem Aufschieliten der Blase, also anf dem Höhepunkt, der Entzündung die Kokken rorhanden seien. Und das nachanweisen ist bisher keinem Untersucher, auch v. Eiselsberg nicht gelungen. Auberdem ist ein bullöses Frysipel im Verhältnis zu den uibrigen eine Seltenheit. Das Erysipel ist eine Infektionskrankheit, und so wenig wie wir bei einer Srhnenscheidenphlegmone, die an eine kleine Verletzung sich anschloli, die Erreger an der Oberfläche der Haut noch nachweisen kïmnen, so wenig gelingt es uns beim Erysipel. Der Erysipelkranke ist also keine (iefahr für seine L'mgebung, aus der unverletaten, erysipelatis erkrankten I Taut gehen keine krankheitserreger hervor. Das ändert sich aber, wenn der Kranke die oft juckende, gespannte: llault anfängt mit seinen Nägeln zn bearbeiten. Durch einen Nagelrils kann das Corium eröffnet werden und das austretende Sekret enthält, wie ich in 2 Fällen nachgewiesen habe, virulente Streptuknkken. IDenn der Nagelritz bedeutet nichts anderes als ein oberflächliches skarificieren der Haut, daher ersetzte ich ihn in meinen Versuchen durch letzteres. Ich habe bis auf einen Fall Haeglers Angaben über diese Art der Untersuchung sonst nicht gefundeu und verfüge selbst nur über 4 Fälle, in denen ich sie angewendet habe. Es sind ties Fall 7 bis 10 und in zweien von ihnen Fall 7 und 10 gelang es mir, einen Streptococcus dabei zu züchten. In Fall 7 war das Wachstum ein reichliches, als ich ihn aus uiner Bouillonkultur isoliert hatte, doch war er nicht virulent für Kaninchen, wenigstens vermochte eine zweimalige Impfung an Kaninchenohr mit verschiedenen Kulturen kein krysipel zu erzeugen. In Fall 10 starb der Stamm ab, als ich ihn weiterzüchten wolte, ehe ich eine Impfung an Kaninchenohr gemacht hatte. Immerhin beweisen die Fälle einwandsfrei, dals durch derartige, die Epidermis durchingende Hautritzchen Streptokokken nach oben dringen können. Auf diese Weise also kann der Kranke Infektionsstoffi an seine Umgebung weitergeben. Denn eimmal kann das eingetrocknete sekret von dem Rils ans in der Luft verteilt werden und aufserdem vermag der infizierte Nagel seinen Streptokokkenvorrat an seine Umgebung abzugeben. Dieses ist aber auch der tinzige Weg; anf dem beim Erysipel Streptokokken den erkrankten Körper verlassen können, vorausgesetzt natürlich, dals keine die Kokken enthaltende Wunde dabei ist. Fs läuft also alles darauf hinaus, den angegebenen Weg der Tnfektion zu sperren. Hat man ess mit einem gebildeten, verständigen Patienten zu tun, so wird 
es nicht schwer halten, ihn mit strenger Mahnung am Berühren des erkrankten Körperteils zu hindern. Um aber allem vorzubeugen, muß man das Erysipel behandeln wie eine secernierende Wunde, also wie eine Streptokokkenphlegmone z. B., und die erkrankten Körperstellen mit einem gut sitzenden, fest abschließenden Verband verdecken. Dann wird auch ein unruhiger: gar delirierender Kranker keine weitere Infektionsgefahr in sich bergen und weder sich ein sogenanntes springendes Erysipel erzeugen - das meines Erachtens allein auf die geschilderte Weise hervorgerufen wird - noch seine Stationsgefährten in Mitleidenschaft ziehen.

Vermag man also den erkrankten Körperteil durch einen Terband sicher abzuschließen, so kann der Kranke ruhig bleiben, wo er ist. Ist man dazu aber nicht imstande, wie das beispielsweise beim Gesichtserysipel, am Perineum etc., der Fall ist, so tut man vielleicht besser, den Kranken bis zum Ablaufe der Entzündungserscheinungen zu isolieren. Sind diese vorüber, ist das Fieber abgefallen, beginnt das Schuppungsstadium, so bildet der Erkrankte nie mehr eine Quelle der Infektion für seine Umgebung.

Es war nicht der Zweck meiner Arbeit, den geheimnisvollen Wegen nachzuforschen, welche die sprungweise, auf möglichst mit aseptisch Operierten belegten Stationen auftretenden Erysipele genommen haben, das kann nur durch genanes Kontrollieren und Erwägen an Ort und Stelle geschehen. Ob man immer so glücklich sein wird wie Klemm, der in dieser Lage zwei nicht bettlägerige Patienten als Infektionsträger erkannte, die er dann eliminieren konnte, - das steht dahin, gewiß aber sind wir durch solche Befunde auf einen der Schleichpfade des krysipels aufmerksam gemacht. -

Mir lag vor allem daran, den Erysipelkranken etwas von dem ihnen anhaftenden Fluch zu nehmen, der sie gleichsam aus der Gemeinschaft mit anderen Leidenden ausstößt. Wir dürfen, ohne unser Gewissen zu belasten, ein Erysipel mit den Hünden berühren einerlei, ob es im frischen Entzündungs- oder im Schuppungsstadium ist, der Inhalt der Blasen beim Lrysipelas bullosum tut uns so wenig Schaden wie der einer Brandblase beispielsweise. Wer würde sich auch nur einen Augenblick besinnen, eine Lymphangitis irgend welchen Ursprungs anzufassen und zu untersuchen? Und doch ist sie eine bakterielle, oft gewiß auch eine streptomykotische Entzündung; pathologisch-anatomisch handelt es sich un völlig gleiche Prozesse, und klinisch finden sich viele Ähnlichkeiten, ja direkte 
Übergänge. Es kommt unter Jucken oder Brennen zu einer Rötung der Haut mit Temperatursteigerung. Man findet bei beiden Formen die Lymphgefäße voller Kokken, eine starke Hyperämie des umgebenden Gewebes, seröse Exsudation, Leukocytenansammlungen etc., man sieht also - eine wesentliche Übereinstimmung. Wird jetzt aber bei dem einen die Diagnose Erysipel gestellt, so verfällt der Ärmste sofort in die Acht, niemand wagt ihn mehr anzutasten, sich seinem Bett zu nähern, und ganz zuletzt erst wendet man sich mit möglichst langen Instrumenten zu ihm, um ihm die notwendigste Hilfe zu erteilen. Diese übertriebene Furcht darf man nach den übereinstimmenden Untersuchungen der weiter oben bereits genannten Autoren, denen ich meine eigenen zufüge, wohl fallen lassen und kann die Rose von demselben Gesichtspunkte aus betrachten wie jede andere Wundinfektionskrankheit, und dann hat man sogar noch ein Übriges getan.

Meinem verehrten Chef, Herrn Professor König, spreche ich auch an dieser Stelle meinen besten Dank für die Anregung zll dieser Arbeit aus.

\section{Literaturverzeichnis.}

1. Fehleisen, Die Ätiologie des Erysipels. Berlin 1883.

2. v. Eiselsberg, Nachweis von Erysipel in der Luft chirurgischer Krankenzimmer. Langenbecks Archiv 1887. Bd. 35.

3. v. Noorden, Über das Vorkommen von Streptokokken im Blut bei Erysipel. Münchn. med. Wochenschr. 1887.

4. Hägler, Die chirurgische Bedeutung des Staubes. Beiträge zur klinischen Chirurgie. Bd. 9.

5. Petruschky, Untersuchungen über Infektion mit pyogenen Kokken. Zeitschr. f: Hygiene. Bd. 17.

6. Derselbe, Entscheidungsversuche zur Frage der Spezifität der Erysipelstreptokokken. Zeitschr. f. Hygiene. Bd. 33.

7. Emmerich, Münchener med. Wochenschr. 1893. Nr. 18-20.

8. Ucke, Ein Beitrag zur Epidemiologie des Erysipels. Centralbl. f. Bakteriologie u. Parasitenkunde. 1897. I.

9. Jordan, Über die Ätiologie des Erysipels und sein Verhältnis zu den pyogenen Infektionen. Münchn. med. Wochenschr. 1901. Nr. 35.

10. Klemm, Über das Verhältnis des Erysipels zu den Streptomykosen, sowie über die Epidemiologie desselben. Grenzgebiete f. Mediz. u. Chir. 1901.

11. Respinger, Untersuchungen über die angebl. Contagiosität des Erysipels. Beitr. f. klin. Chir. 1900. 